\title{
LRP11-AS1 Promotes the Malignant Behavior of Triple Negative Breast Cancer Cells via the MiR-149- 3p/NRP2 Axis
}

Peng Li

Shenzhen University General Hospital

\section{Yu Zeng}

Shenzhen University General Hospital

\section{Yudan Chen}

Shenzhen University General Hospital

\section{Peng Huang}

Shenzhen University

\section{Xinchun Chen}

Shenzhen University

\section{Weidong Zheng ( $\nabla$ zwdin@szu.edu.cn )}

Shenzhen University General Hospital https://orcid.org/0000-0003-4295-1544

\section{Primary research}

Keywords: LRP11-AS1, IncRNA, miR-149-3p, NRP2, breast cancer

Posted Date: October 21st, 2021

DOl: https://doi.org/10.21203/rs.3.rs-987995/v1

License: (9) (i) This work is licensed under a Creative Commons Attribution 4.0 International License. Read Full License 
LRP11-AS1 promotes the malignant behavior of triple negative breast cancer cells via the miR-149-3p/NRP2 axis

Peng $\mathrm{Li}^{1}$, Yu Zeng ${ }^{1}$, Yudan Chen ${ }^{1}$, Peng Huang ${ }^{2}$, Xinchun Chen ${ }^{3}$, Weidong Zheng ${ }^{1,3}, *$.

${ }^{1}$ Department of Laboratory Medicine, Shenzhen University General Hospital.

${ }^{2}$ Marshall Laboratory of Biomedical Engineering, International Cancer Center, School of Biomedical Engineering, Shenzhen University Health Science Center.

${ }^{3}$ Guangdong Provincial Key Laboratory of Regional Immunity and Diseases, Health Science Center, Shenzhen University.

*Corresponding to: zwdin@szu.edu.cn, Tel: 86-755-21839789. No. 1098 Xueyuan Ave, Nanshan District, Shenzhen City, China 518055 


\section{Abstract}

Background: Breast cancer is the most commonly diagnosed cancer in women. Triple negative breast cancer (TNBC) is the most difficult subtype of breast cancer to treat due to the deficiency in drug-targetable receptors. LRP11-AS1, a newly identified oncogenic long noncoding RNA (IncRNA) was found to be significantly overexpressed in TNBC cells. The aim of this study is to investigate the malignant roles and the oncogenic mechanisms of LRP11-AS1 in TNBC.

Methods: CCK-8, colony formation, transwell migration and transwell invasion assays were performed to study the functions of LRP11-AS1. Quantitative PCR and western blot were used to determine the gene expression. Bioinformatics analysis and dual-luciferase reporter assay were conducted to study IncRNA and miRNA interactions.

Results: LRP11-AS1 was found to be significantly overexpressed in TNBC cells compared to the non-TNBC cells and normal mammary epithelial cells. Knockdown of LRP11-AS1 could inhibit the growth and metastasis of TNBC cells and regulate cell cycle. Mechanistically, LRP11-AS1 was found to act as a competing endogenous RNA (ceRNA) to sponge miR-149-3p. Silencing of LRP11-AS1 increased the expression of miR-149-3p and overexpression of miR-149-3p suppressed the expression of LRP11-AS1. Inhibition of miR-149-3p could reverse the anticancer effect of LRP11-AS1 deficiency in TNBC cells. Moreover, Neuropilin-2 (NRP2) was found to be 
the target of miR-149-3p. Rescue experiments revealed that NRP2 overexpression could rescue the anticancer effect of LRP11-AS1 deficiency in TNBC cells.

Conclusion: LRP11-AS1 overexpressed in TNBC showed the oncogenic effects possibly by sponging miR-149-3p and regulating the miR-1493p/NRP2 axis, which indicated LRP11-AS1 as a potential diagnostic biomarker and therapeutic target in TNBC.

Key words: LRP11-AS1, IncRNA, miR-149-3p, NRP2, breast cancer

\section{Background}

Breast cancer is the most frequently diagnosed cancer in women. In 2020, female breast cancer has surpassed lung cancer as the most commonly diagnosed cancer, with an estimation of 2.3 million new cases worldwide [1]. Triple negative breast cancer (TNBC) is a subtype of breast cancer defined by a deficiency in estrogen, progesterone and HER2 receptors. TNBC was highly aggressive with high recurrence and organ metastasis rate. Due to the lack of drug-targetable receptors, chemotherapy is the only recommended systemic treatment of TNBC, inevitably with resistance and relapse. New diagnostic biomarkers and drug targets are needed for the treatment of this type of breast cancer [2, 3].

ENSG00000273132, located at chromosome 6 is a novel long noncoding RNA (IncRNA) antisense to low-density lipoprotein (LDL) receptor related protein 11 (LRP11). This IncRNA was reported to be overexpressed in 
papillary thyroid cancer [4]. In our previous research, we named it LRP11 antisense RNA 1 (LRP11-AS1). LRP11-AS1 was found to promote the growth and metastasis of papillary thyroid cancer by targeting the miR615-3p/Akt2 axis and Akt/mTOR signaling (unpublished results). LncRNAs are long noncoding RNAs functioning as master regulators of gene expression in various biological events and disease processes including cancer. They could regulate the target genes in the transcriptional or posttranscriptional levels [5]. A recent "competing endogenous RNA (ceRNA)" hypothesis proposed that IncRNA could regulate gene expression by sequestering microRNAs (miRNAs) to control their concentration within the cell and act as a negative regulator of miRNAs and therefore, a positive regulator of gene expression [6]. miR-149-3p was reported to be a tumor suppressive miRNA in breast cancer. The expression of miR-149-3p was reported to be decreased in the clinical samples of breast cancer. Inhibition of miR-149-3p could promote the proliferation, invasion and migration of breast cancer cells [7].

Neuropilin-2 (NRP2) is a member of neuropilin family, which are cell surface antigens that bind to vascular endothelial growth factor (VEGF). NRP2 was found to be involved in multiple cellular physiological functions including proliferation, angiogenesis and migration $[8,9]$. It was found to be overexpressed in invasive breast cancer and TNBC, and was correlated with lymph node metastasis $[9,10]$. 
In our previous study, we characterized the functions of LRP11-AS1 and its oncogenic mechanisms in thyroid cancer. LRP11-AS1 was found to promote the growth and metastasis of papillary thyroid cancer cells by targeting the miR-615-3p/Akt2 axis and Akt/mTOR signaling (unpublished results). In our recent study, it was found that LRP11-AS1 was significantly overexpressed in TNBC cells compared to the non-TNBC cells and the normal mammary epithelial cells. The roles of LRP11-AS1 played in TNBC and the possible mechanisms of its oncogenic behavior are investigated here.

\section{Materials and methods}

\section{Cell culture and transfection}

MDA-MB-231, MDA-MB-468 and MCF7 cells were purchased from cell bank of Chinese Academy of Sciences (Shanghai, China). Cells were cultured in RPMI 1640 (Gibco, USA) with 10\% Foetal Bovine Serum (FBS, Bovogen, Australia) and $1 \%$ penicillin-streptomycin at $37^{\circ} \mathrm{C}$ in an incubator in a saturated humidified atmosphere supplied with $5 \% \mathrm{CO}_{2}$. MCF10A was cultured in the same cell culture conditions but with $20 \%$ FBS. Knockdown of LRP11-AS1 was performed by transiently transfected the cells with smart silencer (RIBOBIO, China) using lipofectamine 2000 (Invitrogen) according to the manufacturer's protocol. Smart silencer is a mixture of 3 siRNAs and 3 antisense oligonucleotides. The targeted sequences of siRNAs were 5'-CCAGCAGACCTAGCTGCCA-3', 5'- 


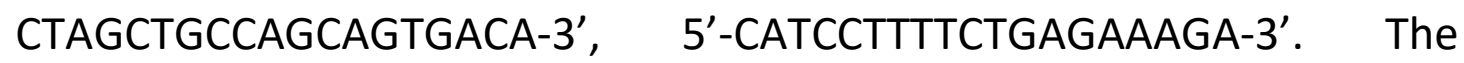
targeted sequences of antisense oligonucleotides were 5'GACAACATGCGTTACCTTCA-3'， 5'-TGCAGCAGTGCCCACTCATA-3'， 5'TGGTCATCTGTGCTCTCGCG-3'. Overexpression of LRP11-AS1 was conducted using lentivirus. The virus were packed with packaging plasmid psPAX2, pMD2.G and PHBLVTM, and recombinant plasmid pHBLV-CMVMCS-EF1-ZsGreen-T2A-Puro encoding the sequence of LRP11-AS1 (ENSG00000273132, Hanbio Biotechnology, China). NRP2 overexpression plasmid (HG10695-CF) and control plasmid (CV012) were purchased from Sino Biological (Beijing, China). miRNA mimics and inhibitors were purchased from RIBOBIO (Guangzhou, China). Cells were transfected using lipofectamine 2000 (Invitrogen) according to the manufacturer's instructions.

\section{Cell proliferation and colony formation assay}

A total of 5000 cells/well were seeded in the 96 -well plate. CCK-8 reagent (Hanbio Biotechnology, China) was used to determine cell proliferation according to the manufacturer's instructions. OD450 was determined at different time points after CCK-8 incubation. Colony formation was performed by seeding 3000 cells/well of MDA-MB-231 or 5000 cells/well of MDA-MB-468 cells in 6-well plate. Cells were allowed to grow for 8 days followed by fixing with methanol and staining with crystal violet.

\section{Migration and invasion assay}


For cell migration assay, $5 \times 10^{\wedge} 4$ cells/well of MDA-MB-231 or $1 \times 10^{\wedge} 5$ cells/well of MDA-MB-468 cells were seeded into the upper chamber of the transwell insert in RPMI medium without FBS. The lower chamber was added with RPMI medium with $10 \%$ FBS. Cells were allowed to migrate for 24h. After that, the cells in the upper chamber of the insert were removed and the migrated cells at the bottom of the insert membrane were fixed with methanol and stained with crystal violet. For cell invasion assay, the membrane of the transwell insert was coated with Matrigel (Corning, 354234 ) according to the manufacturer's instructions. A total of $8 \times 10^{\wedge} 4$ cells/well of MDA-MB-231 cells were seeded in the upper chamber of the insert. Other conditions were the same as cell migration assay.

\section{Western blot}

Cell lysate was prepared with RIPA lysis buffer supplemented with $1 \times$ protease inhibitor (70090050, Biosharp, China) and $1 \times$ phosphatase inhibitor (70080020, Biosharp, China) at $4{ }^{\circ} \mathrm{C}$. Western blot was performed according to the common protocol. The primary antibodies used were anti-cyclin D1 antibody (92G2, Cell Signaling Technology), anti-NRP2 antibody (D39A5, Cell Signaling Technology) and anti-GAPDH (D16H11, Cell Signaling Technology).

RNA extraction, reverse transcription and quantitative PCR (qPCR) Extraction of total RNA and miRNA was conducted using RNA extraction kit (DP419, DP501, Tiangen, China). Reverse transcription of total RNA and 
miRNA was conducted using reverse transcription kits (RR037A, Takara and KR211, Tiangen). qPCR was performed using LightCycler 480 instrument (Roche) and SYBR green qPCR kit (AG11701, Accurate Biology, China). The primers used in the qPCR assays were: LRP11-AS1 forward: 5'CTAGCTGCCAGCAGTGACAA-3', LRP11-AS1 reverse: 5'GCGAGAGCACAGATGACCAC-3'; $\quad \beta$-actin forward: $5^{\prime}$ TGGCATCCACGAAACTACCT-3', $\quad \beta$-actin reverse: $5^{\prime}$ ACGGAGTACTTGCGCTCAG-3'; NRP2 forward: 5'GTGGACCTGCGCTTTTTAACC-3', NRP2 reverse: 5'GCCATTCTGTGTTTCCCTGG-3'. The forward and reversed primers for the quantitation of miR-149-3p was designed and synthesized by Tiangen. The primers for the quantitation of U6 were: U6 forward: 5'AACGCTTCACGAATTTGCGT-3', U6 reverse: 5'- CTCGCTTCGGCAGCACA-3'.

\section{Flow cytometry}

A total of $1 \times 10^{\wedge} 5$ cells/well were seeded in 12 -well plate for $24 \mathrm{~h}$ before transfection with smart silencer for $72 \mathrm{~h}$ followed by cell cycle analysis using cell cycle analysis kit from BD (550825). BD FACSCanto II flow cytometry was used as assay system.

\section{Dual-luciferase reporter assay}

Fragments of LRP11-AS1 containing the binding site of miR-149-3p or the mutant sequence were synthesized and sub-cloned into the pmirGLO dual-luciferase reporter plasmid (GENEWIZ, China). Cells were seeded in 
24-well plate at a density of $5 \times 10^{\wedge} 4$ cells/well for $24 \mathrm{~h}$ before cotransfection with miR-149-3p mimics or mimics NC and the wild type dualluciferase reporter plasmid or the mutant plasmid. Luciferase activity was determined at $48 \mathrm{~h}$ post-transfection using the Dual-Luciferase Reporter Assay System (E1910, Promega). Fragments of the 3'-untranslated region ( $\left.3^{\prime}-U T R\right)$ of NRP2 containing the binding site of miR-149-3p or the mutant sequence were synthesized and sub-cloned into the pmirGLO dualluciferase reporter plasmid (GENEWIZ, China). Other experiment conditions were the same.

\section{Statistical analysis}

All quantitative results were presented as the mean \pm standard deviation (SD). The Student's t-test was used to compare the statistical significance between two groups. Differences were considered significant as ${ }^{*} \mathrm{P}<0.05$; ${ }^{* * P}<0.01, * * * \mathrm{P}<0.001$.

\section{Results}

LRP11-AS1 promoted the growth and metastasis of TNBC cells and regulated cell cycle

In our previous research, LRP11-AS1 was found to be an oncogenic IncRNA in thyroid cancer. The role of LRP11-AS1 in breast cancer was investigated here. As shown in Figure 1A, LRP11-AS1 was found to be overexpressed in TNBC cell line MDA-MB-231 and MDA-MB-468 compared to the non-TNBC cell line MCF7 and the mammary epithelial 
cell line MCF10A. Knockdown of LRP11-AS1 (Figure 1B) inhibited the proliferation of TNBC cells (Figure 1C and 1D). Overexpression of LRP11AS1 (Figure 1E) promoted the proliferation of TNBC cells (Figure $1 \mathrm{~F}$ and 1G). Silencing of LRP11-AS1 inhibited (Figure 1H) whereas overexpression promoted (Figure 1I) the colony formation of TNBC cells. Cell cycle analysis indicated that silencing of LRP11-AS1 inhibited cell cycle G1/S transition of MDA-MB-231 cells (Figure 1J) and inhibited cell cycle S/G2 transition of MDA-MB-468 cells (Figure 1K). The expression of cyclin D1 was downregulated after the knockdown of LRP11-AS1 (Figure 1L and $1 \mathrm{M}$ ). The effect of LRP11-AS1 on the metastasis of TNBC cells was evaluated. Knockdown of LRP11-AS1 inhibited the migration and invasion of TNBC cells (Figure $1 \mathrm{~N}$ ). Overexpression of LRP11-AS1 promoted the migration and invasion of TNBC cells (Figure 10). These results suggested that LRP11-AS1 could promote the growth and metastasis of TNBC cells and also regulate cell cycle.

\section{miR-149-3p was the target of LRP11-AS1 in TNBC}

Bioinformatics analysis was performed to investigate the possible binding target of LRP11-AS1. Among the possible interacting miRNAs predicted by LncRNASNP2 (http://bioinfo.life.hust.edu.cn/IncRNASNP\#!/), miR-149-3p was selected for further study since it was reported as a tumor suppressive miRNA in breast cancer. Figure 2A showed that TNBC cell line MDA-MB-231 and MDA-MB-468 showed significantly lower expression of 
miR-149-3p compared to the mammary epithelial cell line MCF10A. Knockdown of LRP11-AS1 in TNBC cells upregulated the expression of the tumor suppressive miR-149-3p (Figure 2B). Moreover, overexpression of miR-149-3p in TNBC cells suppressed the expression of LRP11-AS1 (Figure 2C). Dual-luciferase reporter assay was performed to investigate the binding between LRP11-AS1 and miR-149-3p. Figure 2D exhibited the possible binding sequence between LRP11-AS1 and miR-149-3p predicted by bioinformatics analysis. Figure $2 \mathrm{E}$ and $2 \mathrm{~F}$ indicated that miR-149-3p could bind to LRP11-AS1 at the predicted binding site. These results indicated that miR-149-3p was a target of LRP11-AS1 in TNBC cells.

Overexpression of miR-149-3p inhibited the growth and metastasis of TNBC cells

The effect of miR-149-3p on the growth and metastasis of TNBC was investigated. miR-149-3p was overexpressed in TNBC cells (Figure 3A). Figure $3 B$ and $3 C$ showed that overexpression of miR-149-3p inhibited the proliferation of TNBC cells. Figure 3D and 3E showed that inhibition of miR-149-3p by inhibitors enhanced the proliferation of TNBC cells. Moreover, overexpression of miR-149-3p inhibited the colony formation (Figure 3F), migration and invasion (Figure 3G) of TNBC cells.

miR-149-3p inhibition reversed the anticancer effect of LRP11-AS1 deficiency in TNBC cells

Rescue experiments were performed to investigate whether LRP11-AS1 
promoted the growth and metastasis of TNBC cells by targeting miR-1493p. Inhibition of miR-149-3p partially reversed the inhibitory effect of LRP11-AS1 deficiency on cell proliferation (Figure 4A and 4B) and colony formation (Figure 4C) in TNBC cells. Figure 4D exhibited that inhibition of miR-149-3p partially restored the inhibitory effect of LRP11-AS1 knockdown on the migration and invasion of TNBC cells. These results indicated that knockdown of LRP11-AS1 inhibited the growth and metastasis of TNBC cells by targeting miR-149-3p.

NRP2 was a target of miR-149-3p and LRP11-AS1 regulated NRP2 through miR-149-3p

The downstream targets of miR-149-3p were predicted using online bioinformatics analysis tool TargetScan (http://www.targetscan.org/vert 72/). Neuropilin-2 (NRP2) was selected as the downstream target of miR-149-3p for further study due to its essential role played in breast cancer. Figure $5 \mathrm{~A}$ showed the relative expression of NRP2 in different cell lines. TNBC cell line MDA-MB-231 and MDA-MB-468 showed higher expression of NRP2 compared to the nonTNBC cell line MCF7 and mammary epithelial cell line MCF10A. Figure 5B represented the possible binding site on the $3^{\prime}$-untranslated region (UTR) of NRP2 to miR-149-3p. Dual-luciferase reporter assay indicated the binding of miR-149-3p to NRP2 at the predicted binding site (Figure 5C and 5D). Overexpression of miR-149-3p downregulated the mRNA (Figure 
$5 E$ ) and protein (Figure 5F and 5G) levels of NRP2 in TNBC cells. Silencing of LRP11-AS1 inhibited the mRNA (Figure $5 \mathrm{H}$ ) and protein (Figure $5 \mathrm{I}$ and 5J) expression of NRP2 in TNBC cells. Rescue experiments indicated that inhibition of miR-149-3p restored the suppression of NRP2 by LRP11-AS1 knockdown in both of the transcriptional (Figure $5 \mathrm{~K}$ and $5 \mathrm{~L}$ ) and translational (Figure $5 \mathrm{M}$ and $5 \mathrm{~N}$ ) levels. These results suggested that NRP2 was a target of miR-149-3p and NRP2 was regulated by LRP11-AS1 through miR-149-3p.

Overexpression of NRP2 reversed the anticancer effect of LRP11-AS1 deficiency in TNBC cells

Since NRP2 was determined to be the target of miR-149-3p and was regulated by LRP11-AS1, rescue experiments were performed by overexpressing NRP2 in TNBC cells. Figure 6A showed the overexpression of NRP2 in MDA-MB-231 cells. The overexpression of NRP2 in MDA-MB468 was insignificant. Overexpression of NRP2 partially rescued the inhibitory effect of LRP11-AS1 deficiency on cell proliferation (Figure 6B). Overexpression of NRP2 also partially rescued the inhibitory effect of LRP11-AS1 deficiency on the colony formation (Figure 6C), migration and invasion (Figure 6D) of TNBC cells. These results indicated that knockdown of LRP11-AS1 inhibited the growth and metastasis of TNBC cells by inhibiting NRP2. LRP11-AS1 that was overexpressed in TNBC cells promoted the growth and metastasis of TNBC cells by targeting the miR- 
149-3p/NRP2 axis.

\section{Discussion}

Triple negative breast cancer (TNBC) is a very aggressive subtype of breast cancer characterized by a deficiency in the hormone receptors, which could serve as drug targets. Chemotherapy is the only recommended systemic treatment with inevitable resistance or relapse. In this study, IncRNA LRP11-AS1 was firstly reported to be overexpressed in TNBC cells compared to the non-TNBC cells and normal mammary epithelial cells. Functional studies suggested that silencing of LRP11-AS1 inhibited the growth and metastasis of breast cancers and induced cell cycle arrest. Mechanistically, miR-149-3p was predicted to be the target of LRP11-AS1 by bioinformatics analysis. miR-149-3p was previously reported to be downregulated in breast cancer clinical samples [7]. In our study, low expression of miR-149-3p was found in TNBC cell lines. Overexpression of miR-149-3p could inhibit the growth and metastasis of TNBC, which indicated the tumor suppressive role of miR-149-3p in TNBC cells. Dualluciferase reporter assay proved the binding of LRP11-AS1 with miR-149$3 p$ in the predicted binding site. More importantly, knockdown of LRP11AS1 increased the expression of miR-149-3p and overexpression of miR149-3p reduced the expression of LRP11-AS1. Rescue experiments showed that inhibition of miR-149-3p could partially rescue the anticancer effect of LRP11-AS1 deficiency in TNBC cells. These results indicated that 
LRP11-AS1 possibly served as the ceRNA to sponge miR-149-3p to promote the growth and metastasis of TNBC.

NRP2 was previously reported as a member of the membrane protein neuropilin family. Increased expression of NRP2 was found in invasive and TNBC and was correlated with lymph node metastasis $[9,10]$. In our study, dual-luciferase reporter assay revealed the interaction between miR-149$3 p$ and the $3^{\prime}-U T R$ of NRP2. miR-149-3p mimics could inhibit the expression of NRP2 in TNBC cells. Moreover, silencing of LRP11-AS1 could also inhibit the expression of NRP2 in TNBC cells. Rescue experiments represented that inhibition of miR-149-3p could restore the inhibited expression of NRP2 by LRP11-AS1 knockdown, indicating that LRP11-AS1 regulated NRP2 expression through miR-149-3p. Functionally, overexpression of NRP2 could partially reverse the anticancer effect of LRP11-AS1 deficiency on the proliferation, migration and invasion of TNBC cells. Figure 7 represented a graphic description of the possible mechanisms of the oncogenic role of LRP11-AS1 played in TNBC. The overexpression of LRP11-AS1 in TNBC lead to the suppression of miR-149$3 p$ and the upregulation of NRP2, which resulted in the promotion of the growth and metastasis of TNBC cells. LRP11-AS1 exhibited the tumorigenic effect in TNBC by sponging miR-149-3p and targeting the miR-149-3p/NRP2 axis. LRP11-AS1 could serve as a potential biomarker in the diagnosis and treatment of TNBC. 


\section{Conclusions}

LncRNA LRP11-AS1, which was overexpressed in TNBC cells could promote the growth and metastasis of TNBC cells by working as a ceRNA to sponge miR-149-3p and regulating the miR-149-3p/NRP2 axis, indicating LRP11AS1 as a potential biomarker in the diagnosis and treatment of TNBC.

\section{Abbreviations:}

TNBC: Triple negative breast cancer; IncRNA: Long noncoding RNA; ceRNA: Competing endogenous RNA; LRP11: Low-density lipoprotein (LDL) receptor related protein 11; LRP11-AS1: LRP11 antisense RNA 1; NRP2: Neuropilin-2; miRNAs: MicroRNAs; 3'-UTR: 3'-untranslated region.

\section{Acknowledgement}

Not applicable.

\section{Authors' contributions}

L.P.: conceptualization of the study, conducting experiments, analyzing data, writing the original manuscript, reviewing and editing. Z.Y. and C.Y.: conducting experiments. H. P. and C. X.: conceptualization of the study and reviewing the manuscript. Z.W.: conceptualization of the study, funding acquisition and reviewing the manuscript.

\section{Funding}

Funding was received from Guangdong Provincial Science and Technology Program (No. 2019B030301009) and Natural Science Foundation of 
Shenzhen University General Hospital (No. SUGH2018QD027).

\section{Availability of data and materials}

The datasets used and/or analyzed during the current study are available from the corresponding author on reasonable request.

\section{Declarations}

\section{Ethics approval and consent to participate}

Not applicable.

\section{Consent for publication}

All authors agreed upon the publication of this manuscript.

\section{Competing interests}

The authors declare that they have no competing interests.

\section{Reference}

1. Sung H, Ferlay J, Siegel RL, Laversanne M, Soerjomataram I, Jemal A, Bray F: Global Cancer Statistics 2020: GLOBOCAN Estimates of Incidence and Mortality Worldwide for 36 Cancers in 185 Countries. CA: A Cancer Journal for Clinicians 2021, 71(3):209-249.

2. Medina MA, Oza G, Sharma A, Arriaga LG, Hernández Hernández JM, Rotello VM, Ramirez JT: Triple-Negative Breast Cancer: A Review of Conventional and Advanced Therapeutic Strategies. Int J Environ Res Public Health 2020, 17(6):2078.

3. Kumar P, Aggarwal R: An overview of triple-negative breast cancer. 
Archives of Gynecology and Obstetrics 2016, 293(2):247-269.

4. Goedert L, Plaça JR, Fuziwara CS, Machado MCR, Plaça DR, Almeida PP, Sanches TP, Santos JFd, Corveloni AC, Pereira IEG et al: Identification of Long Noncoding RNAs Deregulated in Papillary Thyroid Cancer and Correlated with BRAFV600E Mutation by Bioinformatics Integrative Analysis. Scientific Reports 2017, 7(1).

5. Peng $W X$, Koirala $P$, Mo $Y Y$ : LncRNA-mediated regulation of cell signaling in cancer. Oncogene 2017, 36(41):5661-5667.

6. Dykes IM, Emanueli C: Transcriptional and Post-transcriptional Gene Regulation by Long Non-coding RNA. Genomics Proteomics Bioinformatics 2017, 15(3):177-186.

7. Dong Y, Chang C, Liu J, Qiang J: Targeting of GIT1 by miR-149* in breast cancer suppresses cell proliferation and metastasis in vitro and tumor growth in vivo. Onco Targets Ther 2017, 10:5873-5882.

8. Rizzolio S, Tamagnone L: Multifaceted Role of Neuropilins in Cancer. Current Medicinal Chemistry 2011, 18(23):3563-3575.

9. Zhao M, Zhang M, Tao Z, Cao J, Wang L, Hu X: miR-331-3p Suppresses Cell Proliferation in TNBC Cells by Downregulating NRP2. Technol Cancer Res Treat 2020, 19:1-9.

10. Yasuoka H, Kodama R, Tsujimoto M, Yoshidome K, Akamatsu H, Nakahara M, Inagaki M, Sanke T, Nakamura Y: Neuropilin-2 expression in breast cancer: correlation with lymph node metastasis, poor prognosis, 
and regulation of CXCR4 expression. BMC Cancer 2009, 9:220-220. 


\section{Figures}
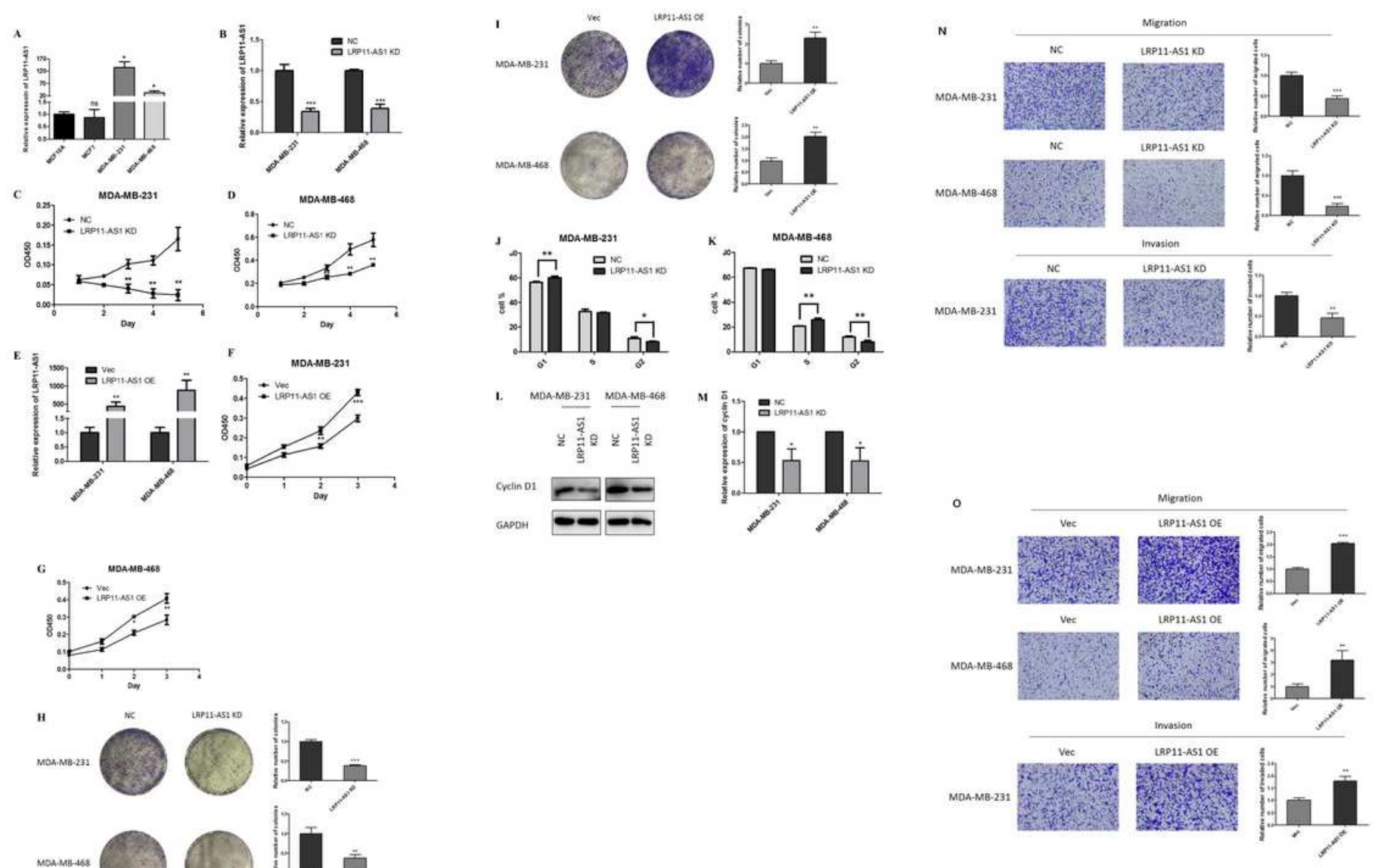

\section{Figure 1}

LRP11-AS1 promoted the growth and metastasis of TNBC cells and regulated cell cycle A, LRP11-AS1 was found to be overexpressed in TNBC cells. The expression of LRP11-AS1 was evaluated by qPCR. B, LRP11-AS1 was knocked down in TNBC cells. The expression of LRP11-AS1 was evaluated by qPCR. C-D, Knockdown of LRP11-AS1 inhibited the proliferation of TNBC cells. CCK-8 assay was performed to determine the proliferation of TNBC cells. E, LRP11-AS1 was overexpressed in TNBC cells. The expression of LRP11-AS1 was evaluated by qPCR. F-G, Overexpression of LRP11-AS1 promoted the proliferation of TNBC cells. $\mathrm{H}$, Silencing of LRP11-AS1 inhibited the colony formation of TNBC cells. I, Overexpression of LRP11-AS1 enhanced the colony formation of TNBC cells. J-K, Knockdown of LRP11-AS1 induced cell cycle arrest of TNBC cells. L-M, Knockdown of LRP11-AS1 downregulated cell cycle checkpoint cyclin D1. Western blot results were shown in $L$ and quantitation of western blots was shown in M. N, Silencing of LRP11-AS1 inhibited the migration and invasion of TNBC cells. 0, Overexpression of LRP11-AS1 enhanced the migration and invasion of TNBC cells. Data were presented as mean $\pm S D$. Statistic significant differences were indicated as $* P<0.05, * \star P<0.01, * \star * P<0.001$. 
A

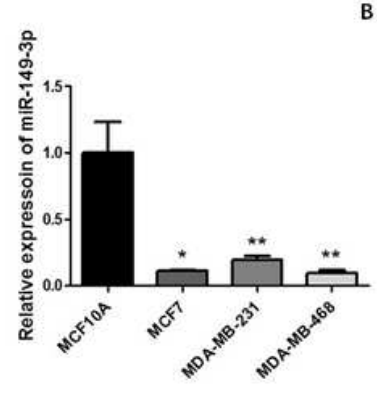

C

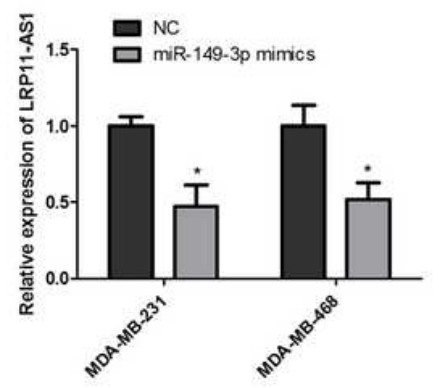

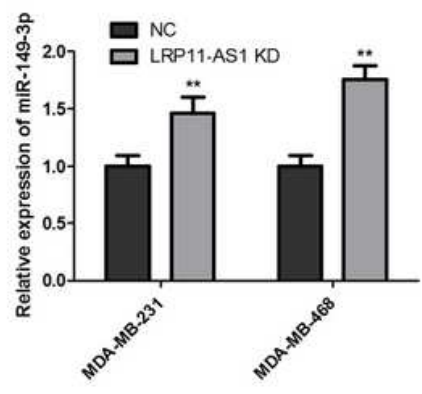

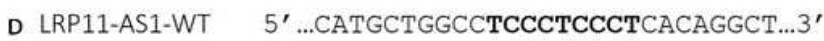<smiles>C1=CC=C1</smiles>

$\operatorname{miR}-149-3 p \quad 3^{\prime}$ CGUGUCGGGGGCAGGGAGGGA 5'

LRP11-AS1-MUT $\quad 5^{\prime}$...CATGCTGGCCAGGGAGGGACACAGGCT...3'

E

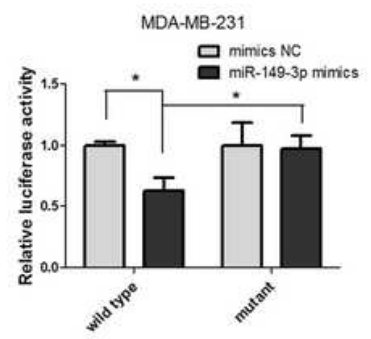

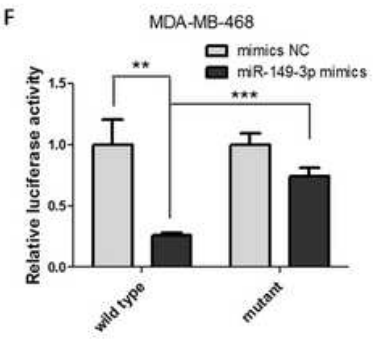

\section{Figure 2}

miR-149-3p was the target of LRP11-AS1 in TNBC A, The expression of miR-149-3p was found to be downregulated in TNBC cells and negatively correlated to LRP11-AS1. The expression of miR-149-3p was evaluated by qPCR. B, Knockdown of LRP11-AS1 increased the expression of miR-149-3p in TNBC cells. C, Overexpression of miR-149-3p decreased the expression of LRP11-AS1 in TNBC cells. D, The predicted binding sequence between LRP11-AS1 and miR-149-3p. E-F, Dual-luciferase reporter assay of the interaction between LRP11-AS1 and miR-149-3p. Cells were co-transfected with miRNA mimics and the dual-luciferase reporter plasmid. Luciferase activity was measured at $48 \mathrm{~h}$ post-transfection. Data were presented as mean $\pm S D$. Statistic significant differences were indicated as $* P<0.05, * \star P<0.01$, $\star * \star P<0.001$. 

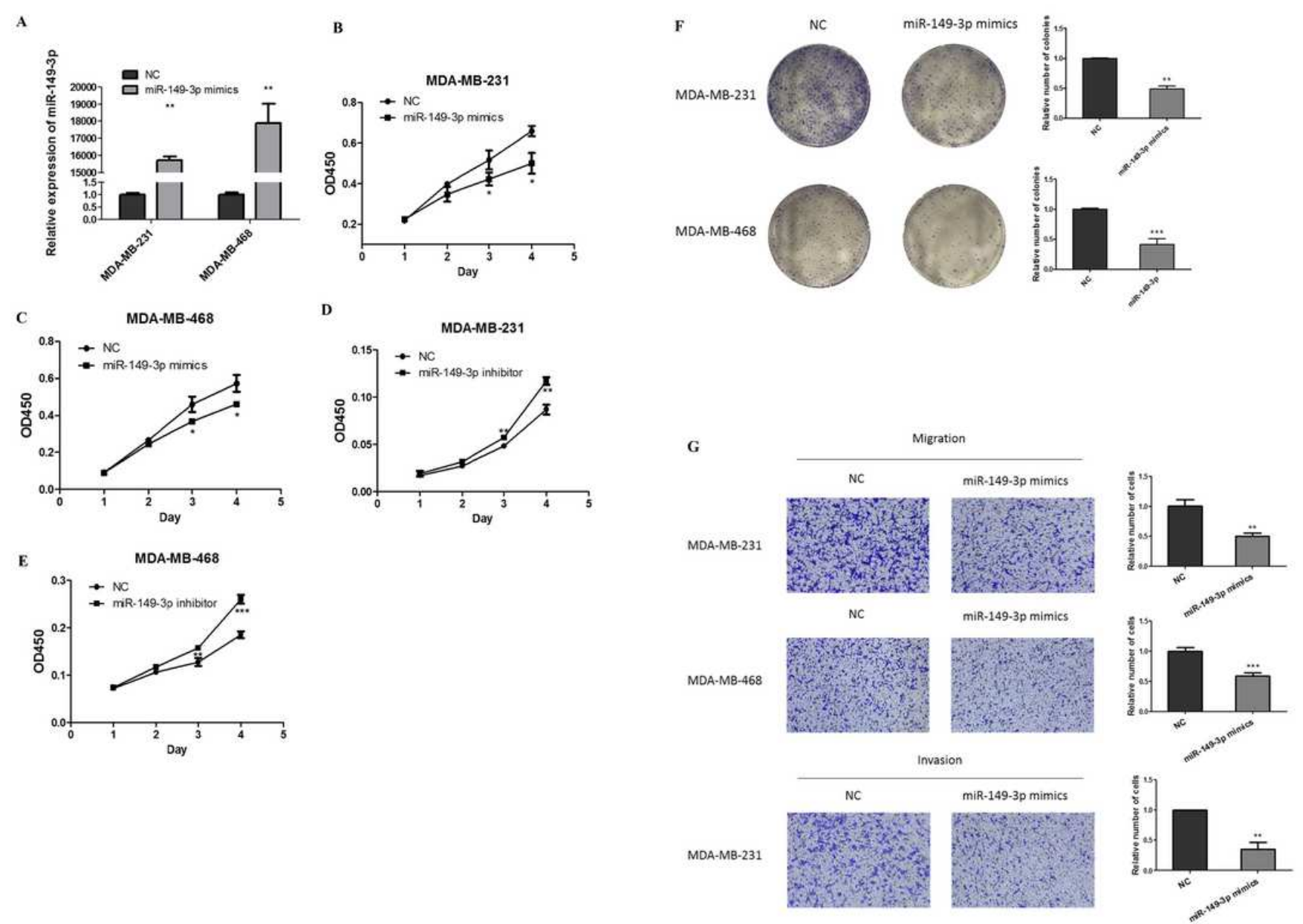

Figure 3

Overexpression of miR-149-3p inhibited the growth and metastasis of TNBC cells A, miR-149-3p was overexpressed in TNBC cells. The expression of miR-149-3p was evaluated by qPCR. B-C, Overexpression of miR-149-3p inhibited the proliferation of TNBC cells. CCK-8 assay was performed to determine the proliferation of TNBC cells. D-E, Inhibition of miR-149-3p promoted the proliferation of TNBC cells. F, Overexpression of miR-149-3p abrogated the colony formation ability of TNBC cells. G, Overexpression of miR-149-3p attenuated the migration and invasion ability of TNBC cells. Data were presented as mean $\pm S D$. Statistic significant differences were indicated as ${ }^{*} P<0.05,{ }^{*} P<0.01, * \star * P<0.001$. 


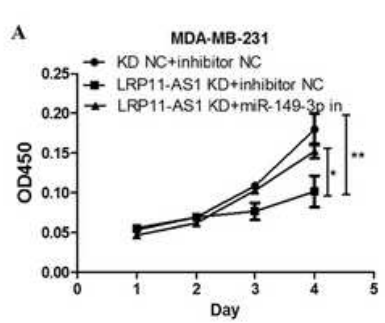

C
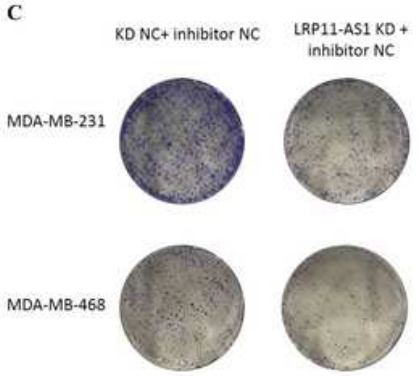

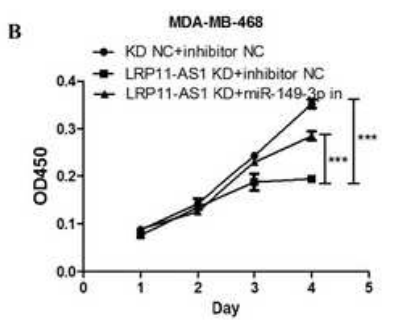

LRP11-AS1 KD + miR-149-3p in
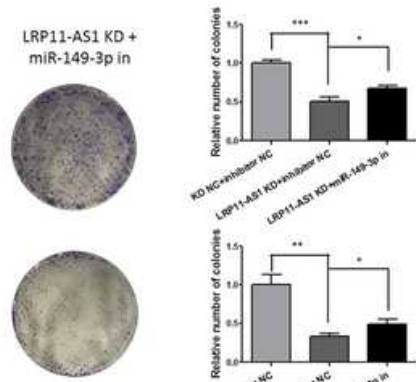

D

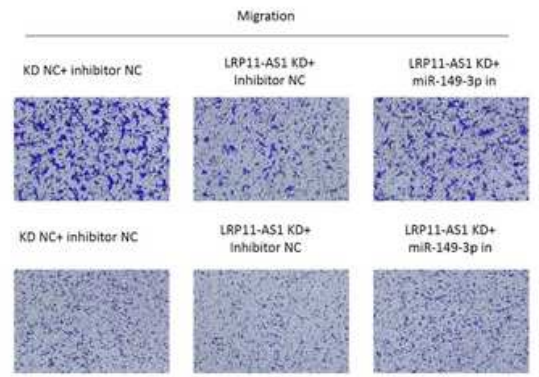

invasion LRP11.ASI KD+

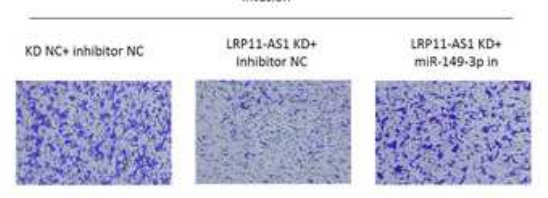

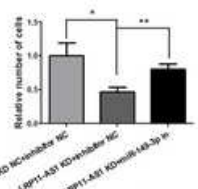
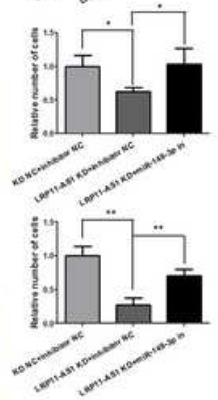

\section{Figure 4}

miR-149-3p inhibition reversed the anticancer effect of LRP11-AS1 deficiency in TNBC cells A-B, miR-149$3 p$ inhibition partially reversed the inhibitory effect of LRP11-AS1 knockdown on the proliferation of TNBC. C, miR-149-3p inhibition partially reversed the inhibitory effect of LRP11-AS1 knockdown on the colony formation of TNBC. D, Inhibition of miR-149-3p partially restored the anticancer effect on the migration and invasion by LRP11-AS1 knockdown. Data were presented as mean \pm SD. Statistic significant differences were indicated as $* P<0.05, * \star P<0.01, * \star * P<0.001$. 

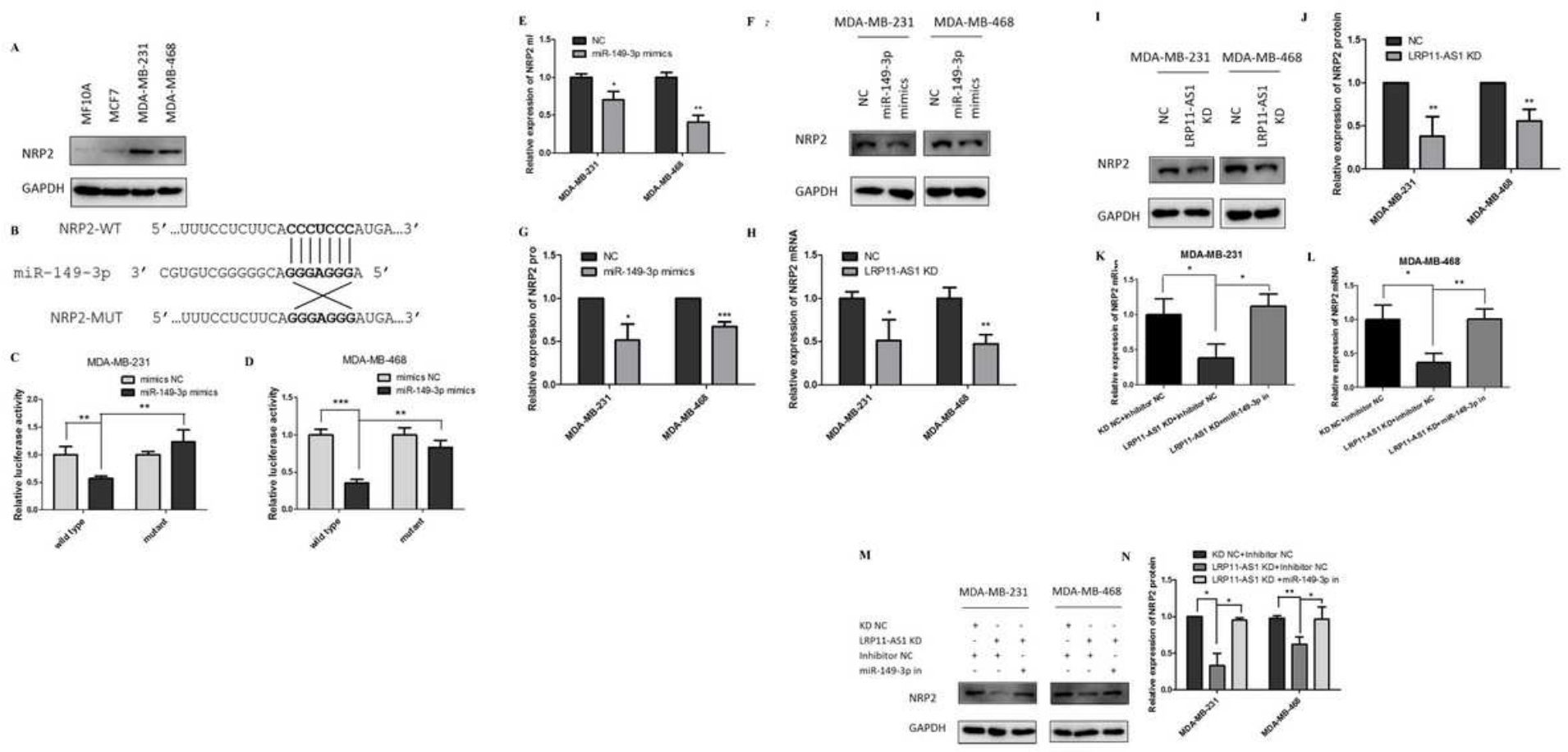

Figure 5

NRP2 was a target of miR-149-3p and LRP11-AS1 regulated NRP2 through miR-149-3p A, NRP2 was found to be overexpressed in TNBC cells compared to the non-TNBC cells and normal mammary epithelial cells. The expression of NRP2 was evaluated by western blot. B, The predicted binding sequence between NRP2 and miR-149-3p. C-D, Dual-luciferase reporter assay of the interaction between NRP2 and miR-149-3p. Cells were co-transfected with miRNA mimics and the dual-luciferase reporter plasmid. Luciferase activity was measured at $48 \mathrm{~h}$ post-transfection. E, Overexpression of miR-149-3p downregulated the expression of NRP2 mRNA. The expression of NRP2 was evaluated by qPCR. F-G, Overexpression of miR-149-3p downregulated the expression of NRP2 protein. The expression of NRP2 was evaluated by western blot $\mathrm{H}$, Knockdown of LRP11-AS1 downregulated the expression of NRP2 mRNA. The expression of NRP2 was evaluated by qPCR. I-J, Knockdown of LRP11-AS1 downregulated the expression of NRP2 protein. The expression of NRP2 was evaluated by western blot. K-L, LRP11-AS1 regulated the expression of NRP2 mRNA through miR-149-3p. The expression of NRP2 was evaluated by qPCR. M-N, LRP11-AS1 regulated the expression of NRP2 protein through miR-149-3p. The expression of NRP2 was evaluated by western blot. Data were presented as mean \pm SD. Statistic significant differences were indicated as $* P<0.05, * * P<0.01, \star \star * P<0.001$. 
A

MDA-MB-231

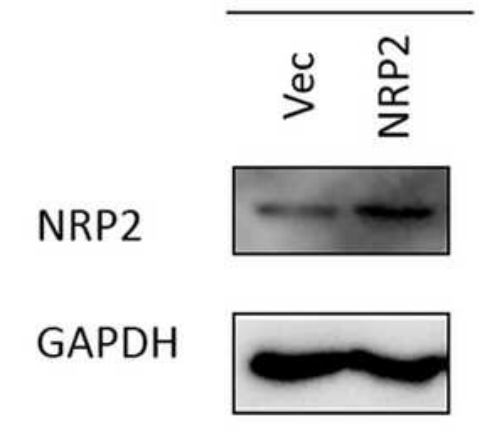

C

MDA-MB-231

KD NC+ OE vec

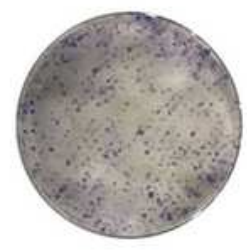

D

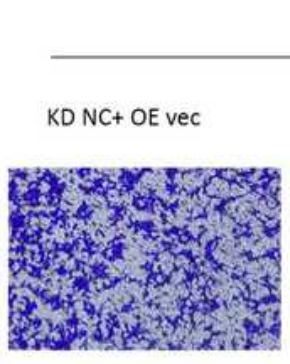

B

\author{
MDA-MB-231
}

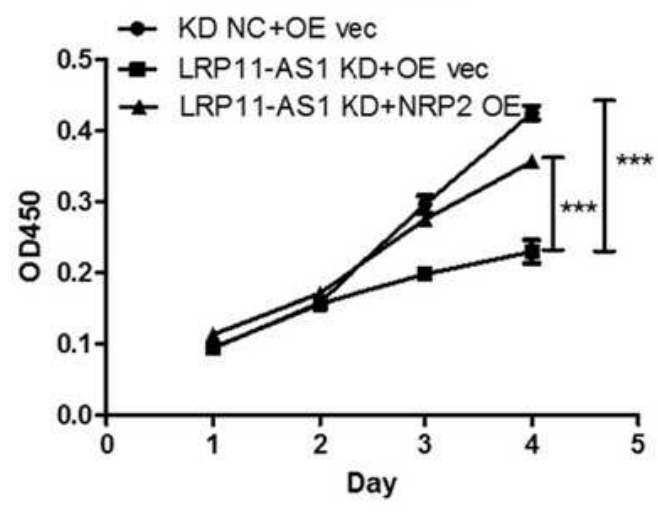

LRP11-AS1 KD +
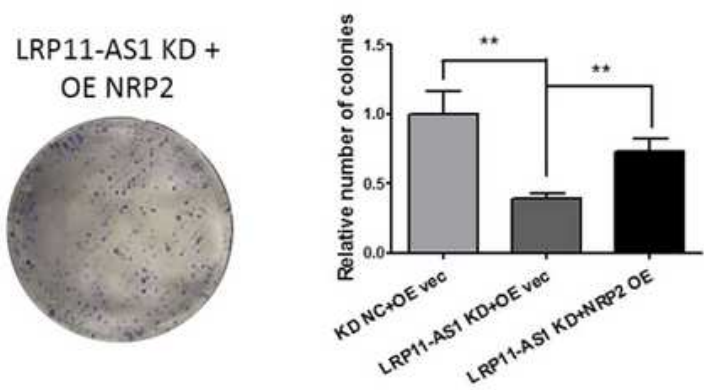

Migration

LRP11-AS1 KD+ OE vec
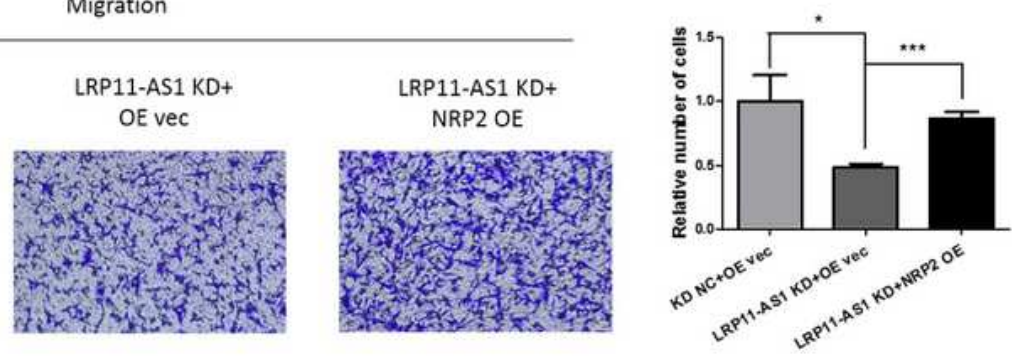

Invasion

LRP11-AS1 KD+ OE vec

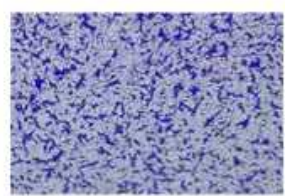

LRP11-AS1 KD+ NRP2 OE

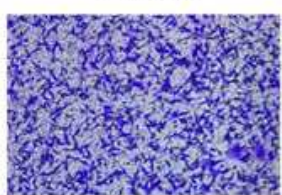

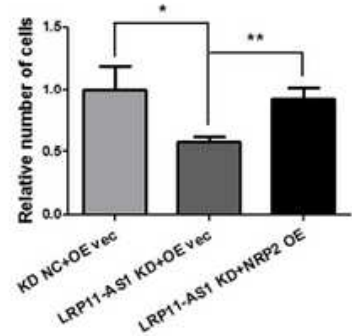

\title{
Figure 6
}

Overexpression of NRP2 reversed the anticancer effect of LRP11-AS1 deficiency in TNBC cells A, Overexpression of NRP2 in TNBC cells. MDA-MB-231 cells were transfected with NRP2 or vector control plasmid. The expression of NRP2 was evaluated by western blot. B, Overexpression of NRP2 partially reversed the inhibitory effect of LRP11-AS1 deficiency on cell proliferation. CCK-8 assay was performed to determine the proliferation of TNBC cells. C, Overexpression of NRP2 partially reversed the inhibition of 
the colony formation by LRP11-AS1 knockdown. D, Overexpression of NRP2 partially reversed the inhibition of the migration and invasion by LRP11-AS1 knockdown. Data were presented as mean \pm SD. Statistic significant differences were indicated as $* P<0.05,{ }^{*} \mathrm{P}<0.01,{ }^{* \star *} \mathrm{P}<0.001$.

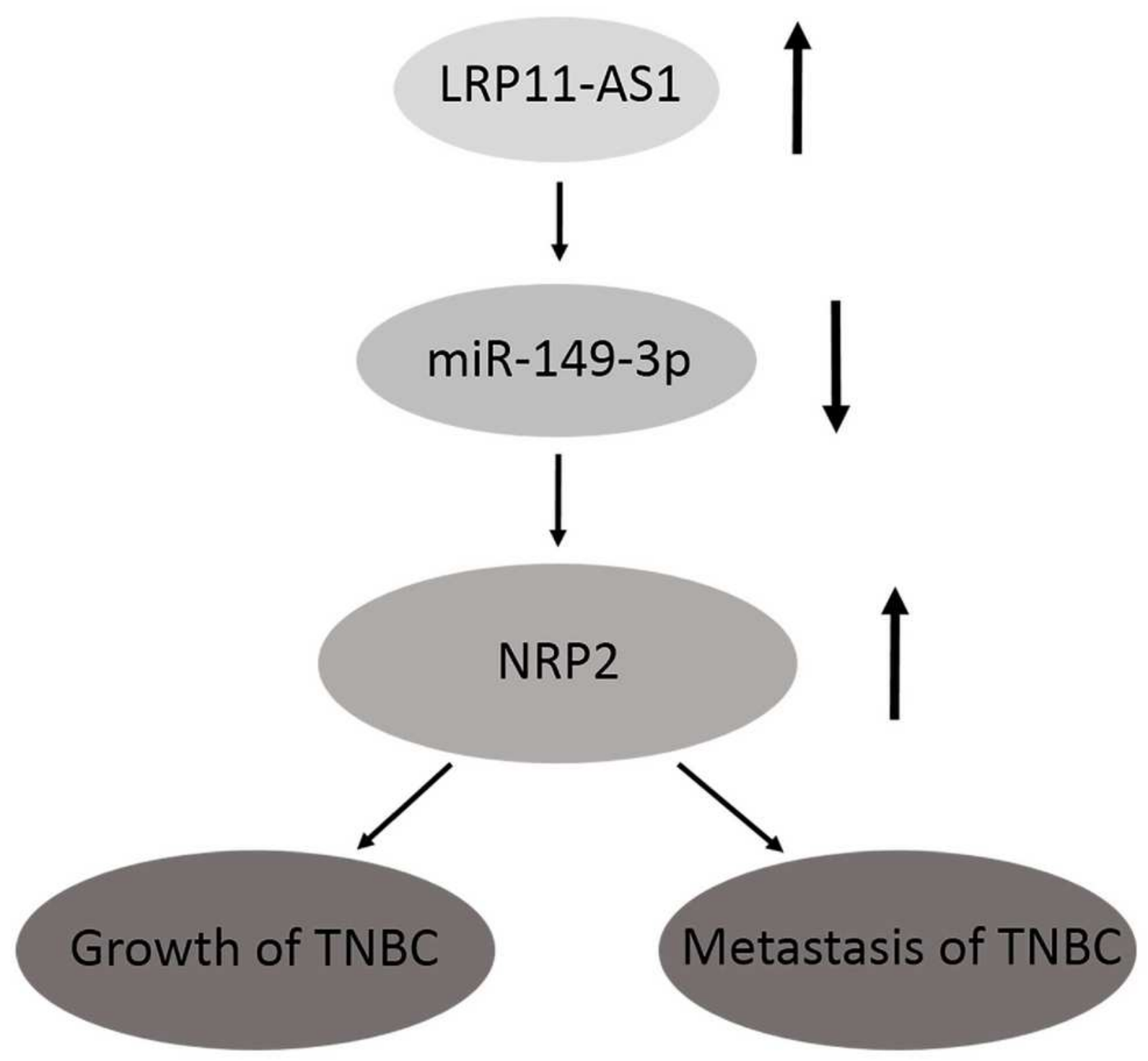

Figure 7

LRP11-AS1 promoted the growth and metastasis of TNBC cells by targeting the miR-149-3p/NRP2 axis. A graphic description of the mechanisms underlying the oncogenic role of LRP11-AS1 in TNBC cells. The overexpression of LRP11-AS1 in TNBC suppressed the expression of miR-149-3p and therefore, enhanced the expression of NRP2, which resulted in the promotion of the growth and metastasis of TNBC cells. LRP11-AS1 exhibited the malignant behavior in TNBC by sponging miR-149-3p and regulating the miR149-3p/NRP2 axis. 